Original Paper http://ajol.info/index.php/ijbcs http://indexmedicus.afro.who.int

\title{
Effets des composts ménagers sur les propriétés du sol et sur la productivité des cultures légumières : cas de la tomate (Lycopersicon esculentum Mill)
}

\author{
Joseph TSHALA UPITE ${ }^{1 *}$, Alain KITABALA MISONGA ${ }^{1}$, \\ Emery KASONGO MUKONZO LENGE ${ }^{2}$ et Luciens NYEMBO KIMUNI ${ }^{3}$
}

${ }^{1}$ Faculté des Sciences Agronomiques, Université de Kolwezi (UNIKOL), République Démocratique du Congo.

${ }^{2}$ Unité de recherche en Evaluation des Terres et Agro météorologie, Département de Gestion de Ressources Naturelles et Renouvelables, Faculté des Sciences Agronomiques, Université de Lubumbashi B.P 1825, Lubumbashi, RD Congo.

${ }^{3}$ Faculté des Sciences Agronomiques, Université de Lubumbashi, Lubumbashi, RD Congo. *Auteur correspondant ; E-mail : tshalajoseph@gmail.com; Tel : (+243)976050952

\section{RESUME}

A Kolwezi, les sols sont généralement de texture sableuse. Leurs richesses chimiques se situent à un niveau très bas pour tous les éléments nutritifs avec une incidence directe sur les rendements des cultures. Afin d'évaluer l'impact du compost ménager sur la restauration de la fertilité des sols maraîchers de Kolwezi, un essai conduit en split-plot avec deux variétés de tomate (Tanya et Tengeru) et trois doses (20, 25 et 30 t.ha $\left.^{-1}\right)$ de compost ménager appliquées seules et/ou combinées à $250 \mathrm{~kg} \mathrm{ha}^{-1}$ de NPK en comparaison d'un témoin sans fertilisation a été installé dans deux sites maraîchers (Musompo et Mwangeji). Les déchets collectés auprès de 100 ménages ont été triés et pesés en vue de déterminer la composition physique d'une poubelle et la part biodégradable a été compostée. Pour renseigner la valeur agronomique du compost, l'analyse chimique a été effectuée. De cette étude, il a été révélé qu'en moyenne, une poubelle à Kolwezi est composée de plus de $50 \%$ de déchets dégradables dont le compost qui en est issu est un réservoir de $\mathrm{N}, \mathrm{P}, \mathrm{K}$ et autres éléments indispensables à la culture. Appliquées sur un sol perçu pauvre et confirmé par les analyses chimiques, les doses de compost améliorent la fertilité de sol consécutivement le rendement de la tomate. 11,4 t.ha ${ }^{-1}$ contre 11,7 t.ha ${ }^{-1}$ obtenu respectivement aux apports de $30 \mathrm{t}^{\mathrm{h}} \mathrm{ha}^{-1}$ de compost et $30 \mathrm{t}^{\mathrm{h}} \mathrm{ha}^{-1}+250 \mathrm{~kg} \cdot \mathrm{ha}^{-1}$ de NPK, suppose que le compost issu des déchets ménagers serait utilisé en légume-culture sans être combiner aux engrais minéraux. L'application supplémentaire de $250 \mathrm{~kg}$ NPK induit inévitablement une baisse de la profitabilité ainsi, 20 t.ha $^{-1}$ de compost est recommandable. L'analyse microbienne et l'usage de compost ménager sur les légumes feuilles seraient une seconde phase de l'étude.

(C) 2019 International Formulae Group. All rights reserved.

Mots clés : Compost de déchet ménager, restauration de sol, maraichères, gestion, Kolwezi.

\section{Effects of household composts on soil properties and productivity of vegetable crops: the case of tomato (Lycopersicon esculentum Mill)}

\begin{abstract}
In Kolwezi, the soils are generally sandy in texture. Their chemical richness is at a very low level for all nutrients with a direct impact on crop yields. In order to assess the impact of household compost on restoring the fertility of market garden soils in Kolwezi, a split-plot trial with two tomato varieties (Tanya and
\end{abstract}




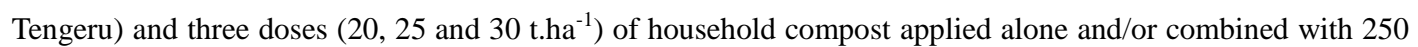
$\mathrm{kg} \mathrm{ha}^{-1}$ of NPK compared to a control without fertilizer was set up in two market garden sites (Musompo and Mwangeji). The waste collected from 100 households was sorted and weighed to determine the physical composition of a bin and the biodegradable part was composted. To inform the agronomic value of the compost, chemical analysis was carried out. From this study, it was revealed that on average, a bin in Kolwezi is composed of more than $50 \%$ degradable waste, from which the compost that is produced is a reservoir of $\mathrm{N}$, $\mathrm{P}, \mathrm{K}$ and other elements essential for cultivation. Applied on a soil perceived as poor and confirmed by chemical analyses, the compost doses improve soil fertility as a result of the tomato yield. 11.4 t.ha $^{-1}$ against 11.7 t.ha $^{-1}$ obtained respectively with the inputs of 30 t.ha- 1 of compost and 30 t.ha $^{-1}+250 \mathrm{~kg}$.ha- 1 of NPK, supposes that the compost from household waste would be used in legume-cultivation without being combined with mineral fertilizers. The additional application of $250 \mathrm{~kg}$ NPK inevitably leads to a decrease in profitability, so $20 \mathrm{t}^{-h a^{-1}}$ of compost is recommended. Microbial analysis and the use of household compost on leafy vegetables would be a second phase of the study.

(C) 2019 International Formulae Group. All rights reserved.

Keywords: Household waste compost, soil restoration, market gardening, management, Kolwezi.

\section{INTRODUCTION}

Les espaces (péri) urbains à Kolwezi
sont caractérisés par une importante croissance démographique avec comme conséquence l'accroissement de la ville. Toutefois, cette dynamique urbaine n'est pas accompagnée d'une mise en place d'infrastructures, des services de base ou d'une politique adéquate de gestion des déchets, fait qui a conduit à une augmentation criante du volume des déchets ménagers. Cependant, cette dynamique urbaine n'est pas accompagnée par une mise en place d'infrastructures, des services de base ou d'une politique adéquate de gestion des déchets, fait qui a conduit à une augmentation criante du volume des déchets ménagers. Bien plus, cette urbanisation ne s'accompagne non seulement par la production des déchets, elle s'associe aussi à l'accroissement des besoins alimentaires (Ahmed et Ali, 2006; Sotamenou, 2010). Pour produire suffisamment et nourrir cette population sans cesse croissante, plusieurs auteurs dont notamment Mufind et al. (2016), Mondjalis et al. (2017) et Tshibingu et al. (2017) préconisent l'usage des engrais chimiques. Cependant, le coût élevé de ces engrais ne permet pas généralement de compenser les dépenses engagées. Bien plus, les sols de Kolwezi par leurs faibles taux des colloïdes, malgré la fertilisation aux engrais chimiques, l'azote et le potassium sont exposés à l'érosion hydrique. Par ailleurs, bien que figure parmi les activités socio-économiques les plus importantes de la ville, l'agriculture maraichère dans et aux alentours de Kolwezi reste confrontée à l'exploitation minière et à l'accroissement de la ville avec l'occupation foncière des terres agricoles (Kitabala et al., 2016), ce qui conduit aux mauvaises pratiques d'utilisation des terres. Combinée aux processus pédologiques naturels, cette situation aboutit à des sols fortement dégradés et très acides sur lesquels la production des principales cultures, légumières notamment, enregistre une baisse de rendement au fil du temps (Kitabala et al., 2016). Selon d'autres écrits, l'apport de fumier pourrait être un moyen efficace et peu onéreux pour restaurer la productivité des sols (Ognalaga et al., 2015), malheureusement ces intrants sont limités à cause de leur faible disponibilité (Lekasi et al., 2003). Dans ce contexte, il devient impérativement important de rechercher les alternatifs soulagements notamment l'utilisation des ressources disponibles dont les biodéchets pour une production à faible coût et durable. En absence de plans adaptés et de législation visant la gestion écologiquement rationnelle des déchets dans la ville de Kolwezi d'une part et, d'un côté à la productivité limitée de sols, le compost en maraîchage comme source des nutriments est un moyen peu onéreux de recycler les déchets (Useni et al., 2013 ; 2014) d'une part et de l'autre part, l'utilisation de compost de déchets ménagers en agriculture est l'une des voies peu onéreuse de la restauration de la fertilité des sols (Serémé et 
Phal, 2007; Mulaji, 2011; El Herradi et al., 2014; Toundou et al., 2014; Useni et al., 2014 ; Baboy et al., 2015). Outre la régénération des propriétés physico-chimiques des sols dégradés soulignée par plusieurs auteurs ailleurs et dans d'autres villes de la République Démocratique du Congo, les composts des déchets ménagers auraient d'autres avantages tels que la réduction des effets serres par séquestration de carbone, la diminution de la masse, du volume de déchets dans les villes (Houot, 2002) et la réduction d'utilisation exclusive des engrais chimiques. Malheureusement, aucunes informations n'existent à ce sujet sur la ville de Kolwezi, alors que les ménages et les pouvoirs publics peinent à gérer les déchets d'une part et d'autre part l'indisponibilité des matières fertilisantes parait l'une des contraintes en légumeculture. En ce sens Kolwezi, constitue un cas idéal pour ces investigations.

Le but de cette recherche est d'évaluer les effets des apports de compost issu des déchets ménagers sur la fertilité des sols maraîchers de Kolwezi et à quelle dose selon les sites. Plus spécifiquement, il s'agira (i) d'apprécier la valeur agronomique de compost, (ii) d'évaluer son impact sur les propriétés chimiques du sol, le rendement et la rentabilité économique en culture de tomate. Cette étude vérifie les hypothèses selon lesquelles : (i) le compost des déchets ménagers permettrait de couvrir le besoin de la tomate en éléments nutritifs équivalant à l'engrais minéral, (ii) le rendement de la tomate et l'amélioration de propriétés chimiques des sols seraient fonction de la variation des doses des composts appliquées, de leur combinaison à l'engrais minéral et du contexte des sites maraîchers et (iii) la rentabilité économique serait fonction des apports des composts appliqués seuls ou combinés aux engrais minéraux.

\section{MATERIELS ET METHODES Milieu d'étude}

Deux sites où les activités maraichères sont beaucoup pratiquées, à savoir Musompo $\left(10^{\circ} 48^{\prime} \mathrm{S}\right.$ et $\left.025^{\circ} 33^{\prime} \mathrm{E}\right)$ et Mwangeji $\left(10^{\circ} 43^{\prime} \mathrm{S}\right.$ et $025^{\circ} 31^{\prime} \mathrm{E}$ ) dans le périurbain de la ville de Kolwezi en République Démocratique du
Congo, ont constitué les sites de cette étude. Kolwezi tire son existence à l'exploitation du cuivre et du cobalt. La ville est aussi un centre bancaire et d'exploitation artisanale. Cette dernière reste l'une des activités économiques les plus courantes dans et autour de Kolwezi (Dibwe, 2006). Kolwezi jouie d'un climat tropical du type $\mathrm{Cw}$ selon le système de classification de Koppen, comprenant deux saisons, une saison sèche (mai à septembre) et une saison pluvieuse (novembre à mars) avec deux mois de transition (avril et octobre) (Malaisse, 1974). Sur le plan floristique, Kolwezi est dominée par la forêt claire "miombo" comportant un couvert arboré discontinu qui domine un tapis de hautes herbes (Kikufi et Lukoki, 2008). Cependant, à cause d'une part des besoins accrus en charbon de bois et de l'autre part, à la pratique d'une agriculture sédentaire, cette végétation a accusé une régression remarquable entre la période allant de 2002 à 2015 (Cabala et al., 2017). Les terres cultivables sont représentées par les Ferrasols, généralement acides, peu fertiles et pauvres en matière organique (Mukalay, 2016). Les expérimentations ont été installées sur des parcelles cultivées pendant plusieurs années sans interruption mais dans un système en rotation (choux-auberginemaïs). Par ailleurs, les mauvaises herbes les plus fréquentes du milieu sont l'Imperata cylindrica (L.) Räusch) (herbe sanglante), Pennisetum purpureum Schumach. (Fausse canne à sucre), Acanthospermum australe (Loefl) Kuntze, Cynodon dactylon (L.) Pers (herbe des Bermudes) et Hyparrhenia rufa (Nees) Stapf.

\section{Nature, préparation et analyse chimique du sol et des composts}

Les déchets collectés auprès de 100 ménages dans toutes les deux communes de la ville, ont été triés à la main selon leur nature (Ghrabi et al., 2002) et la part biodégradable a été compostée sur une durée de 4 mois (Mulaji, 2011). Afin de déterminer la fertilité $\mathrm{du}$ sol, les échantillons ont été prélevés suivant la méthode décrite par (Rouessac et al., 2004) sur chaque site et les analyses ont été réalisées au laboratoire Agro-pédologique de la Faculté des Sciences Agronomiques de 
l'Université de Lubumbashi. Les analyses du sol ainsi que du compost ont portés sur le carbone organique dosé par la méthode de Walkley et Black (1934) alors que la teneur en matière a été obtenue à partir du carbone organique, en utilisant le coefficient multiplicateur 1,724 (Rouessac et al., 2004); sur l'azote par la méthode de Kjeldahl (Mulaji, 2011), le phosphore dosé par colorimétrie suivant la méthode de Schell (Rouessac et al., 2004), le calcium et le potassium dosés par photométrie à flamme (Rouessac et al., 2004) et enfin le $\mathrm{pH}_{\text {eau }}$ mesuré au moyen d'un pH-mètre dans un rapport sol-solution eau de 1:5.

\section{Culture test}

La tomate (Lycopersicon esculentum Mill), une source de revenus non négligeable aux maraîchers a été retenue pour cette étude. Les semences sont issues des variétés (Tanya et Tengeru) produites par le centre SAFARI Kenya, extension de Lubumbashi. Ces variétés ont été retenues pour leur potentiel élevé en rendement (3-35 t.ha ${ }^{-1}$ en milieu naturel) et une bonne résistance au nématode, à la mosaïque virale et au flétrissement bactérien (Minja et al., 2011).

\section{Conduite des essais}

L'essai a été mis en place sur deux sites en 2016 suivant un dispositif expérimental en split-plot à trois répétitions. Deux variétés (Tanya et Tengeru) ont été prises comme facteur principal et trois doses $(20,25$ et 30 t.ha ${ }^{-1}$ ) de compost ménager appliquées seules et/ou combinées à $250 \mathrm{~kg} \mathrm{ha}^{-1}$ de NPK et un témoin sans fertilisation ont été considérés comme deuxième facteur. Après délimitation $\mathrm{du}$ terrain, toutes les opérations en rapport avec la préparation du terrain ont été effectuées à la main, notamment le labour et le hersage.

Les écartements ont été de $0,50 \mathrm{~m}$ entre les lignes et de $0,40 \mathrm{~m}$ sur les lignes, ramenant à une densité de 50.000 plantes.ha $^{-1}$. En vue d'homogénéiser les substrats, l'épandage du compost a eu lieu deux semaines avant transplantation à une profondeur de $10 \mathrm{~cm}$ (Bevacqua et Mellano, 1993), suivi d'un retournement superficiel tandis que l'irrigation était effectuée par simple arrosage, à raison de deux passages par semaine avant la transplantation. L'épandage de NPK a été réalisé 15 jours avant l'apparition de boutons floraux suivant les suggestions de (Elattir et al., 2003). Dans le contexte de l'essai, $250 \mathrm{~kg}$ de NPK correspond à $1 / 4$ de la dose recommandée (Elattir et al., 2003). Les soins d'entretien ont consisté au sarclage et au binage à l'aide d'une houe. Le rythme des arrosages était de deux fois par jour au début de la transplantation avec une séquence de deux jours ; par contre il a été réduit à un arrosage par jour à partir de 15 jours après la transplantation. Afin d'éviter le contact direct des fruits avec le sol, le tuteurage a été effectué au quarante cinquième jour après repiquage. Les paramètres observés au cours de cette étude sont la taille des plantes, le diamètre des tiges tandis qu'à la récolte, qui était par ailleurs échelonnée, les observations ont porté sur le nombre de fruits par plant, le poids de fruits ainsi que le rendement en fruits. Des analyses économiques ont été aussi réalisées. Les charges de la collecte de déchets, le transport, l'achat NPK et le coût d'épandage des engrais minéraux et de compost ont été prises en considération pour évaluer les bénéfices. Le changement de propriété du sol a été évalué par rapport à la concentration initiale de l'élément dans le sol et après récolte par la formule suivante (Kaho et al., 2011):

$$
X(\%)=100 * \frac{X_{2}-X_{1}}{X_{1}}
$$

$\checkmark$ Où : $\mathrm{X}_{1}$ est la valeur du paramètre considéré au début de l'essai

$\checkmark \mathrm{X}_{2}$ est la valeur du paramètre considéré à la fin de l'expérimentation.

Selon la formule, les valeurs négatives suggèrent les pertes des propriétés du sol par contre, les valeurs positives suggèrent une amélioration de la fertilité de sol (Tableau 3).

\section{Analyses statistiques}

Afin de déceler l'influence des composts sur les caractéristiques agronomiques, les données collectées ont été 
soumises à une analyse de variance (ANOVA) suivie de la comparaison des moyennes par le test de Tukey au seuil de probabilité de $5 \%$ à l'aide du logiciel $\mathrm{R}$ version 2.14.0 ${ }^{\circledR}$. Par contre, afin de dégager l'impact d'apport supplémentaire de l'engrais minéral au compost, la comparaison par paires de moyennes a été réalisée par le test $\mathrm{t}$ à une probabilité de 5\%. L'estimation de la rentabilité économique liée à chaque dose pour chaque variété a été obtenue en faisant le rapport entre le revenu net et les coûts totaux.

\section{RESULTATS}

\section{Composition physique d'une poubelle}

Il ressort de la Figure 1 que les plastiques, les métaux, les verres ainsi que la part biodégradable se présentent à des taux différents. Ainsi, il est à noter qu'en moyenne une poubelle d'un ménage à Kolwezi renferme $53 \%$ de matières biodégradables contre $47 \%$ de matières non biodégradables.

\section{Caractérisation chimique du compost}

Les valeurs observées dans le Tableau 1 , attestent que le compost à base d'ordures ménagères serait une source des éléments qui pourraient combler les besoins des plantes en production légumière. Cependant, comparé aux composts obtenus dans d'autres pays et/ou villes, il existe des variabilités en éléments majeurs, sur le $\mathrm{pH}$, le carbone organique et la teneur de la matière organique mais ces valeurs restent comprises dans la gamme prescrite des normes standards (FAO et AFNOR).

Se référant à la norme congolaise, la concentration du $\mathrm{Cu}, \mathrm{Pb}, \mathrm{Zn}, \mathrm{Fe}$ et $\mathrm{Mn}$ dans le compost reste inférieure au seuil d'appréciation d'un bon compost. Autrement dit, son application n'aura pas d'impact négatif sur la qualité de sol ainsi que la croissance des plantes. Ainsi, il contient respectivement 0,$03 ; 0,02 ; 0,023 ; 1,63$ et 0,03 $\%$ (Tableau 2).

\section{Diagnostic et état agro-pédologique des sols des sites étudiés : influence des composts}

Les résultats sur le $\mathrm{pH}$ à la fin de l'expérimentation montrent des taux de variations positifs sur toutes les parcelles fertilisées au compost. Par contre dans les parcelles non amendées, la valeur du $\mathrm{pH}$ diminue davantage par rapport à la valeur observée au débit de la campagne. Des taux de variations négatifs du carbone organique ainsi que de la matière organique sont observés sur le sol de Musompo tandis que sur le site Mwangeji, ces pertes sont importantes seulement sur les parcelles sans amendement organique. Par contre, une tendance d'augmentation positive a été observée dans toutes les parcelles fertilisées (Tableau 3).

De l'observation du tableau 3, il revient à noter que le taux de carbone organique ainsi que le taux de matière organique varient indépendamment des doses et sont meilleures à l'application de 20 t.ha $^{-1}$ de compost. A la fin de l'expérimentation, l'azote, le phosphore et le calcium sont les trois éléments qui ont présenté des taux de changement importants et surtout très élevés dans leur combinaison aux engrais chimiques. Par contre, malgré la richesse du compost en potassium, cela n'a pas empêché la déficience de celui-ci dans tous les deux sites. Les taux de variations négatives obtenus avec le cuivre, le fer, manganèse et sur les valeurs du rapport carbone-azote mettent en évidence encore une fois de plus la capacité du compost à régénérer les propriétés chimiques du sol.

\section{Efficacité du compost d'ordure ménagère} sur la croissance et rendement de la tomate

Les effets des apports croissants de compost ménager sur la croissance et productivité de la tomate sont présentés à la Figure 2. Comparée au traitement témoin, la croissance des plantes est peu affectée par les doses de compost ( $p>0,05$ ). Il ressort des résultats des rapports taille des plantes/diamètre au collet tel que illustrés par la Figure 2 que les plantes se développent plus en hauteur qu'en diamètre (rapport $>1$ ). Par contre, le nombre de fruits produits par plant varie de 3 à 4,9 et les quatre doses de composts peuvent être classées en trois groupes dont 25 et 30 t.ha $^{-1}$ sont les doses ayant produit le plus grand nombre de fruits (> 4 fruits/plant) pendant que les traitements 
sans compost ont été les moins productifs $(<3$ fruits/plant $) \quad(\mathrm{p}<0,05)$. L'application des composts a permis d'augmenter le poids de fruit qui est ainsi passé de 32,1 à 67,4 g. Quant au rendement, il augmente proportionnellement aux doses de compost apportées et reste faible sur les parcelles témoins $(\mathrm{p}<0,05)$.

Les résultats de la Figure 3 montrent que l'effet conjoint des deux engrais améliore les rendements de la tomate à la supplémentation de $250 \mathrm{~kg}$ de NPK à 20 et 25 t.ha ${ }^{-1}$ de compost $(\mathrm{p}<1 \%)$, excepté à la supplémentation $250 \mathrm{~kg}$ de NPK à 30 t.ha $^{-1}$ de compost où les rendements similaires ont été obtenus. L'application complémentaire de 250 $\mathrm{kg}$ de NPK améliore de manière positive ( $\mathrm{p}<$ $1 \%$ ) le poids des fruits, par contre, la croissance des plantes et le nombre des fruits ne varient forcement pas lorsqu'il y a ajout complémentaire des engrais chimiques.

Quant à la réponse variétale, il ressort de la figure 4 qu'il n'existe pas de différences significatives entre ces deux variétés sur le poids de fruits, le nombre des fruits et le rapport taille/diamètre des plantes excepté, le rendement qui varie d'une variété à une autre $(\mathrm{p}=0,042)$. Ainsi, le rendement est de 7,4 t.ha ${ }^{-1}$ avec la variété Tanya et 9,9 t.ha $^{-1}$ avec la variété Tengeru.
De la lecture de la Figure 5, il est ressorti que les rendements de la tomate ont été confondus quel que soit le site d'étude. Autrement dit, il n'y a pas eu de différences significatives sur le rendement et poids de fruits $(p>0,05)$. Par contre, la croissance et le nombre de fruits ont varié significativement d'un site à l'autre $(\mathrm{p}<0,1 \%)$.

\section{Rentabilité économique des composts en culture de tomate}

Le coût total pour un hectare avec l'apport de 20 t.ha $^{-1}$ de compost sans engrais chimique revient à 380.000 Franc congolais (Fc), alors qu'une tonne de tomate revient à \pm 712.962,962 Franc congolais (Fc) sur les marchés de Kolwezi. Globalement, l'application de compost ainsi que leur combinaison aux engrais chimiques ont un effet bénéfique, car les gains de production qu'ils occasionnent ont permis de couvrir toutes les dépenses de production en culture de tomate (Figure 6). Cependant, au regard des effets de chaque traitement tel qu'illustré par la Figure 6, il revient à noter que pour chaque dose de compost, l'apport des engrais minéraux se traduit par une réduction de bénéfice net, autrement dit l'usage du compost seul se révèle être les traitements le plus avantageux.

Tableau 1 : Comparaison des teneurs en éléments majeurs (\%) dans le compost obtenues à Kolwezi aux valeurs obtenues ailleurs et aux normes standards (FAO et AENOR).

\begin{tabular}{lcccccccc}
\hline Pays (ville) & pH & CO & MO & N & C/N & P & K & Ca \\
\hline R.D. Congo (Kolwezi) $^{\text {a }}$ & 7,40 & 7,15 & 12,33 & 0,57 & 13 & 39,50 & 0,69 & 1,70 \\
R.D. Congo (Kinshasa) $^{\mathrm{b}}$ & 7,91 & 27,02 & - & 2,7 & 10 & 0,56 & 0,67 & 3,42 \\
Burkina Faso (Bobo-Dioulasso) $^{\mathrm{c}}$ & 8,88 & 4,01 & 6,91 & 0,33 & 12,4 & 28,65 & 0,00169 & 0,02 \\
Burkina Faso (Bobo-Dioulasso) $^{\mathrm{d}}$ & 7,30 & 4,07 & 7,01 & 0,36 & 11,30 & 27,6 & 1,26 & - \\
Maroc (Rabat) $^{\mathrm{e}}$ & 8,75 & 12,62 & 21,76 & 1,26 & 10,63 & 3,04 & 3,88 & - \\
Algérie (Mostaganem) $^{\mathrm{f}}$ & 6,2 & 27,6 & - & 1,40 & 20 & - & - & - \\
Cameroun (Dschang) $^{\mathrm{g}}$ & 8,32 & 10,45 & 20,90 & 1,22 & - & 1,12 & 2,27 & 0,38 \\
Benin (Abomey Calavi) $^{\mathrm{h}}$ & 7,80 & 1,50 & - & 0,09 & 15,81 & - & - & - \\
FAO, 2005b, $^{\mathrm{i}}$ & & & & 0,5 & $15-20$ & - & $0,4-2,3$ & - \\
AFNOR $^{\mathrm{J}}{ }$ & & & & $>0,025$ & - & 0,3 & $>1$ & - \\
\hline
\end{tabular}

Légende : a: cette étude; b: (Mulaji, 2011); c; (Yé, 2007); d: (Compaoré et al., 2010); e: (El Heradi et al., 2014); f: (Guermoud et Addou, 2014); g: (Tengoua, 2013); (Topanou, 2012) ; i: (FAO, 2005). 


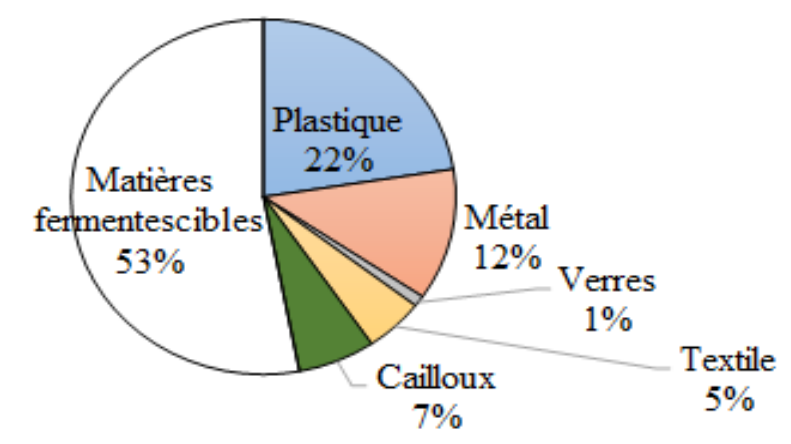

$\square$ Plastique $\square$ Métal $\square$ Verres $\square$ Textile $\square$ Cailloux $\square$ Matières fermentescibles

Figure 1 : Caractéristique physique d'ordures ménagères à Kolwezi.

Tableau 2 : Teneurs en éléments traces métalliques (\%) dans l'échantillon du compost ménager à Kolwezi.

\begin{tabular}{llllll}
\hline Echantillon & Fe & Mn & Zn & Cu & Pb \\
\hline Compost & 1,63 & 0,03 & 0,023 & 0,03 & 0,02 \\
\hline Norme Congolaise \% & & & 0,1 & 0,03 & 0,06 \\
\hline
\end{tabular}

Tableau 3 : Taux (\%) de changement des propriétés des sols des deux sites étudiés, en fonction des traitements appliqués.

\begin{tabular}{|c|c|c|c|c|c|c|c|c|c|c|c|c|}
\hline Site & Traitement & pH & $\mathrm{CO}$ & MO & $\mathbf{N}$ & $\mathbf{C} / \mathbf{N}$ & $\mathbf{P}$ & $\mathbf{K}$ & Ca & $\mathbf{F e}$ & Mn & $\mathbf{C u}$ \\
\hline \multirow{7}{*}{ 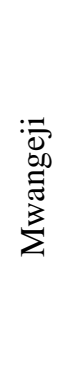 } & T0 & $-7,2$ & $-32,0$ & $-32,0$ & 41,7 & $-50,0$ & 9,9 & $-18,3$ & 23,1 & 2,7 & $-28,3$ & $\mathrm{Nd}$ \\
\hline & $\mathrm{T} 1$ & 1,9 & 160,0 & 160,1 & 66,7 & 62,5 & 45,4 & $-10,2$ & 54,2 & $-14,2$ & $-32,4$ & 64,3 \\
\hline & $\mathrm{T} 4$ & 2,6 & 96,0 & 96,1 & 100,0 & 63,3 & 52,5 & $-28,7$ & 130,8 & $-25,4$ & $-42,2$ & 92,9 \\
\hline & $\mathrm{T} 2$ & 4,1 & 80,0 & 80,1 & 25,0 & 40,6 & 59,6 & $-57,7$ & 84,6 & $-8,4$ & $-23,2$ & 3,6 \\
\hline & $\mathrm{T} 5$ & 1,1 & 112,0 & 112,1 & 292,0 & 10,4 & 95 & $-14,8$ & 46,2 & $-19,8$ & $-41,4$ & $-21,4$ \\
\hline & T3 & 9,5 & 120,0 & 119,9 & 33,3 & $-41,5$ & 17 & $-22,6$ & 153,9 & $-24,3$ & -42 & 7,1 \\
\hline & T6 & $-14,1$ & 16,0 & 16,01 & 133,0 & $-48,2$ & 91,5 & $-14,4$ & 76,9 & $-22,8$ & -32 & 142,9 \\
\hline \multirow{7}{*}{ 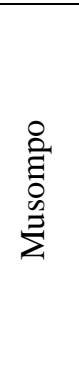 } & T0 & $-1,0$ & $-4,4$ & $-4,5$ & 113,0 & $-80,7$ & 8,4 & $-6,58$ & $\mathrm{Nd}$ & $-6,1$ & 7,3 & 23,1 \\
\hline & $\mathrm{T} 1$ & 17,8 & $-17,9$ & $-17,9$ & 325,0 & $-22,9$ & 30,8 & $-13,9$ & $\mathrm{Nd}$ & $-0,4$ & $-30,7$ & $\mathrm{Nd}$ \\
\hline & $\mathrm{T} 4$ & 3,8 & 44,5 & 44,5 & 600,0 & $-35,7$ & 53,2 & $-8,73$ & $\mathrm{Nd}$ & $-22,4$ & $-4,17$ & $-12,8$ \\
\hline & $\mathrm{T} 2$ & 7,6 & 4,4 & 4,4 & 87,5 & $-89,5$ & 23,3 & $-6,83$ & $\mathrm{Nd}$ & $-5,7$ & 14,9 & 0,0 \\
\hline & T5 & 9,4 & $-26,7$ & $-26,7$ & 25,0 & $-34,2$ & 68,1 & $-10,6$ & $\mathrm{Nd}$ & $-20,0$ & 5,7 & $-23,1$ \\
\hline & T3 & 12,8 & $-17,9$ & $-17,9$ & 62,5 & $-74,3$ & 53,2 & $-19,3$ & $\mathrm{Nd}$ & $\begin{array}{l}-14,3 \\
\end{array}$ & $-4,4$ & $-25,6$ \\
\hline & T6 & 8,6 & 22,2 & 22,2 & 375,0 & $-100,0$ & 34,5 & $-20,3$ & $\mathrm{Nd}$ & $-14,6$ & $-15,1$ & 17,9 \\
\hline
\end{tabular}

Nd: non déterminé, T0: témoin; T1: 20 t.ha $^{-1}$, T2: 25 t.ha $^{-1}$, T3: 30 t.ha $^{-1}$, T4: 20 t.ha $^{-1}+250$ kg NPK, T5: 25 t.ha ${ }^{-1}+250$ kg NPK et T6: 30 t.ha ${ }^{-1}+250$ kg NPK. 

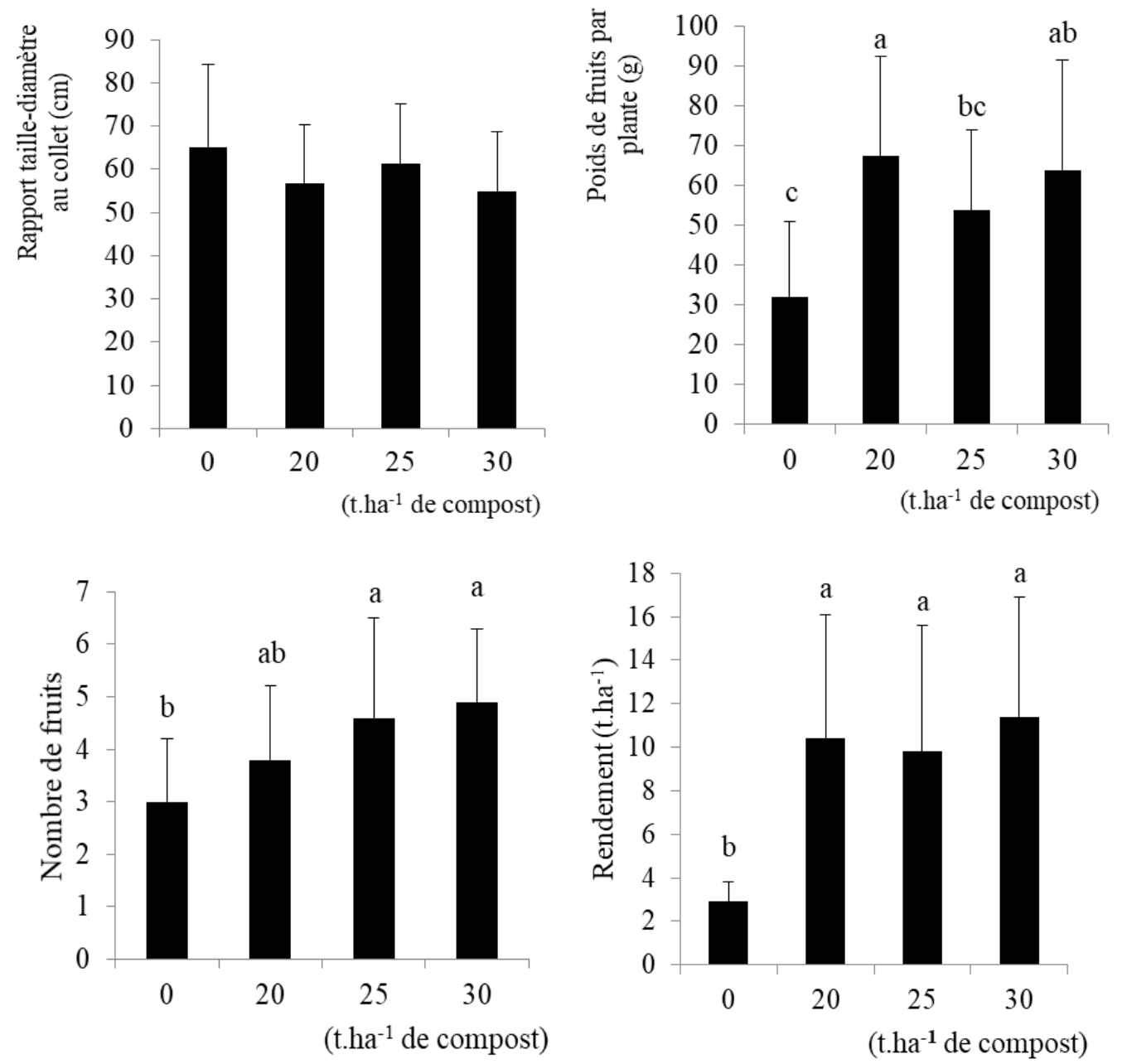

Légende : Les bâtons portant les différentes lettres sont statiquement différents suivant le test de Tukey à la probabilité de 0,05 .

Figure 2 : Effet de doses croissantes de compost sur la croissance et la production de la tomate. 

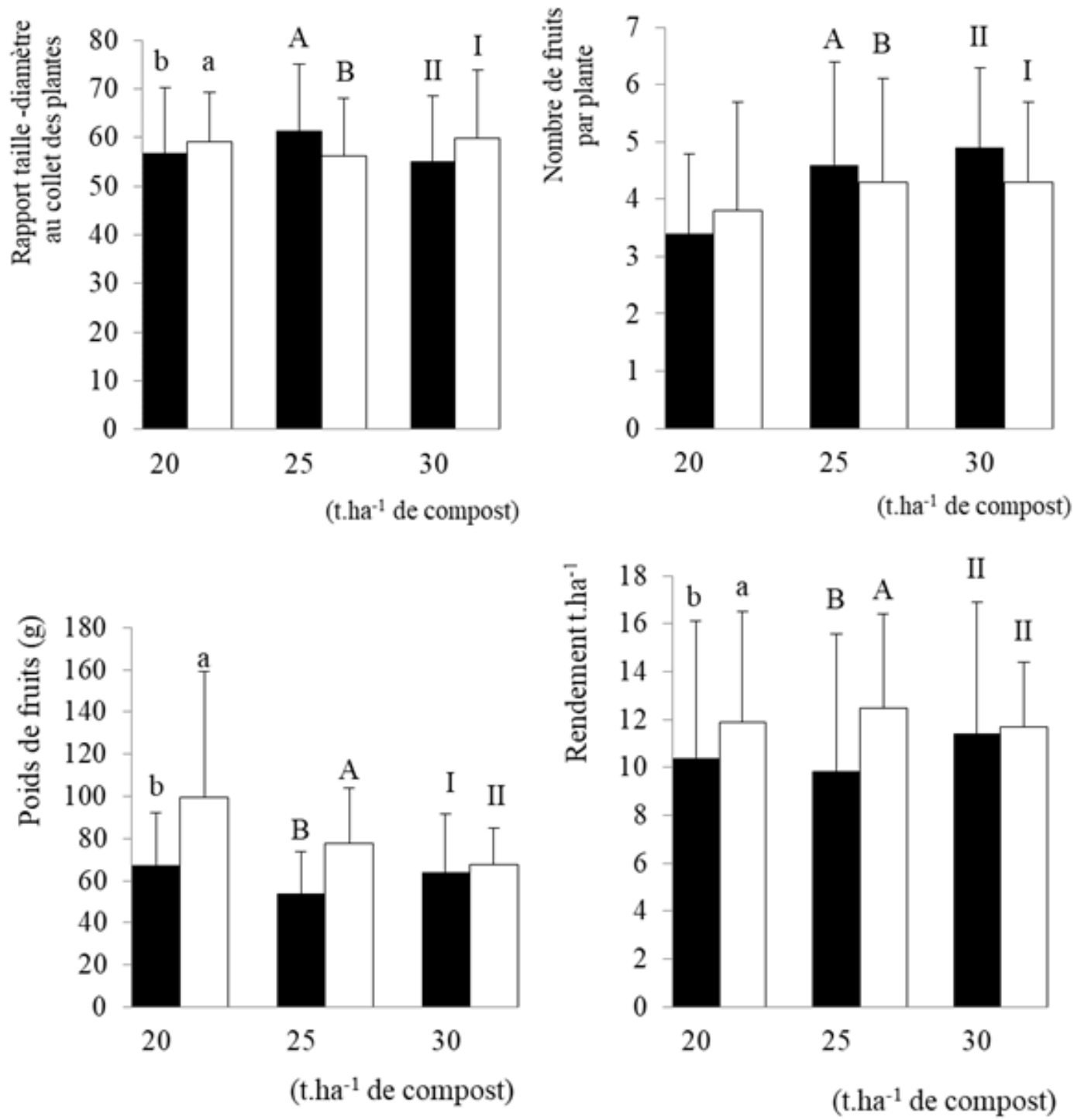

Légende : Le blanc correspond à l'ajout d'un supplément de $250 \mathrm{~kg} \mathrm{NPK}$. Les moyennes ne portant pas les lettres ne sont pas significativement différentes selon le test $\mathrm{t}$ à la probabilité de 0,05 . (a) : symbolise 20 t.ha $^{-1}$ de compost et leur combinaison aux engrais minéraux; (A) symbolise 25 t.ha $^{-1}$ de compost et leur combinaison aux engrais minéraux et (I) symbolise 30 t.ha ${ }^{-1}$ de compost et leur combinaison aux engrais minéraux.

Figure 3: Effet synergique du compost et des engrais minéraux sur la croissance et le rendement de la tomate. 

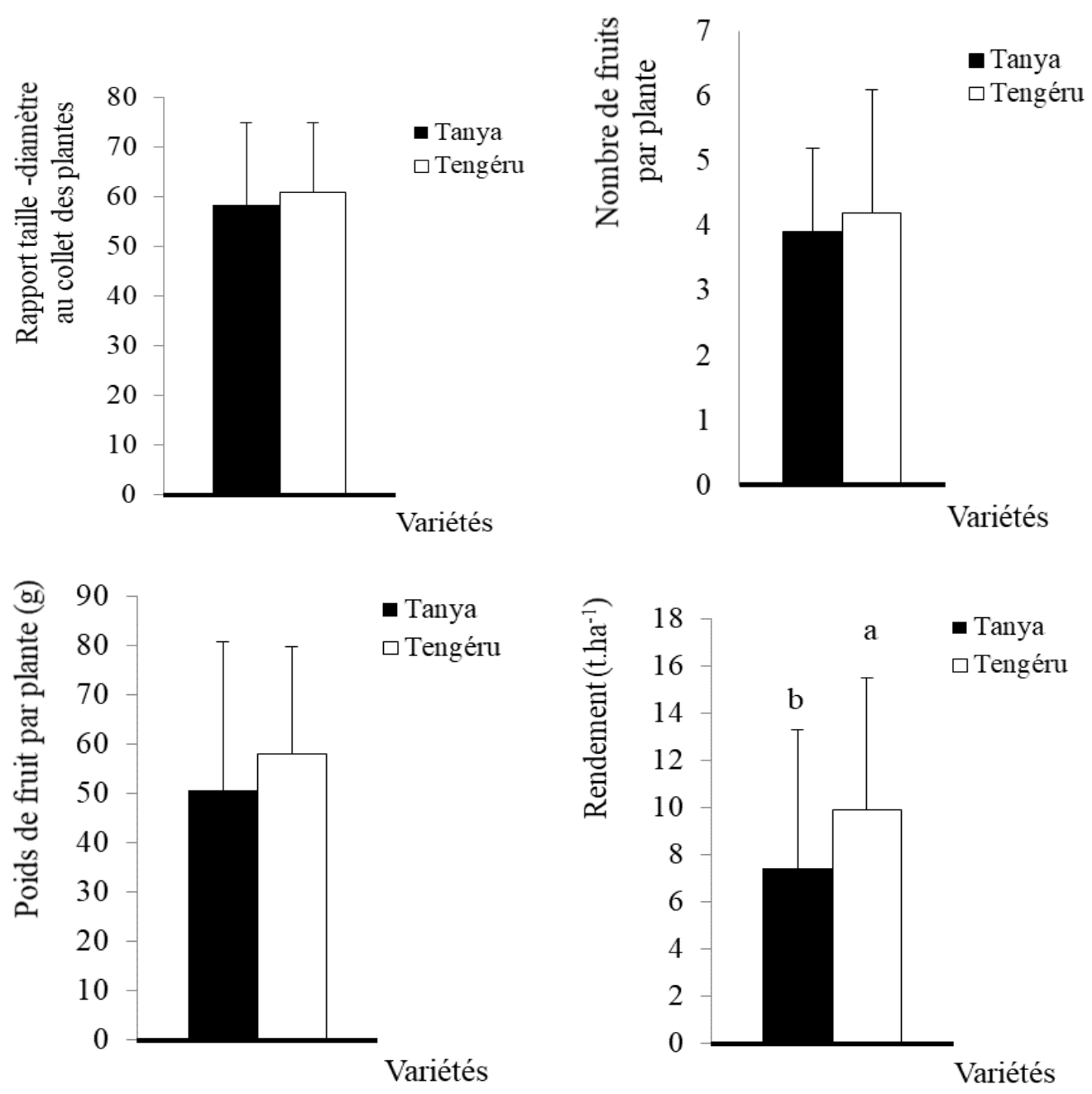

Légende: Les moyennes de cultivars de tomate ne portant pas les lettres ne sont significativement différentes à $\mathrm{P}<0,05$ suivant le test de Tukey à la probabilité de 0,05 .

Figure 4 : Croissance et production de deux variétés (Tanya et Tenguru). 

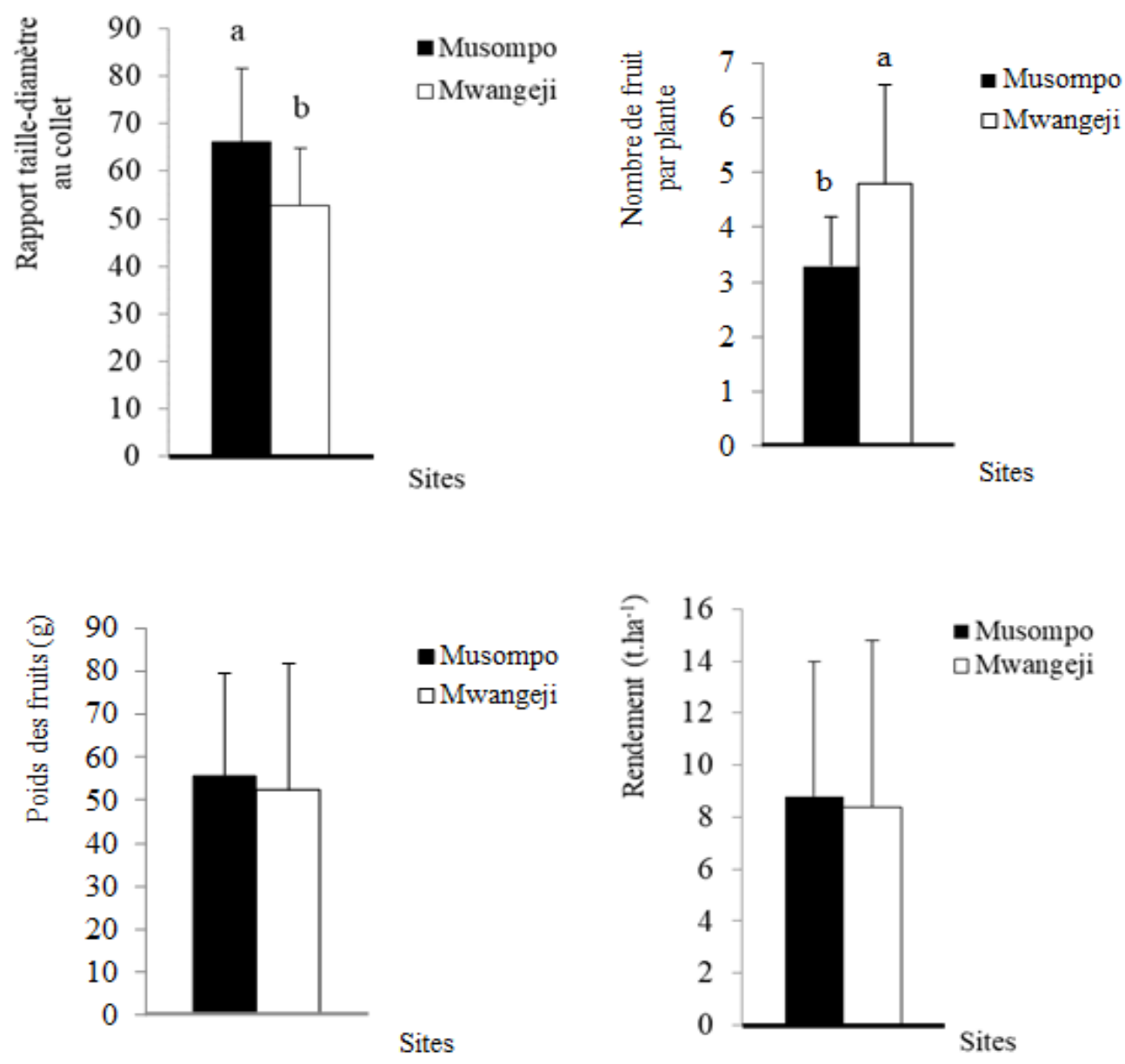

Légende: Les moyennes de cultivars de tomate ne portant pas les lettres ne sont significativement différentes à $\mathrm{P}<0,05$ suivant le test de Tukey à la probabilité de 0,05 .

Figure 5: Croissance et production de la tomate sur les deux sites étudiés (Musompo et Mwangeji).

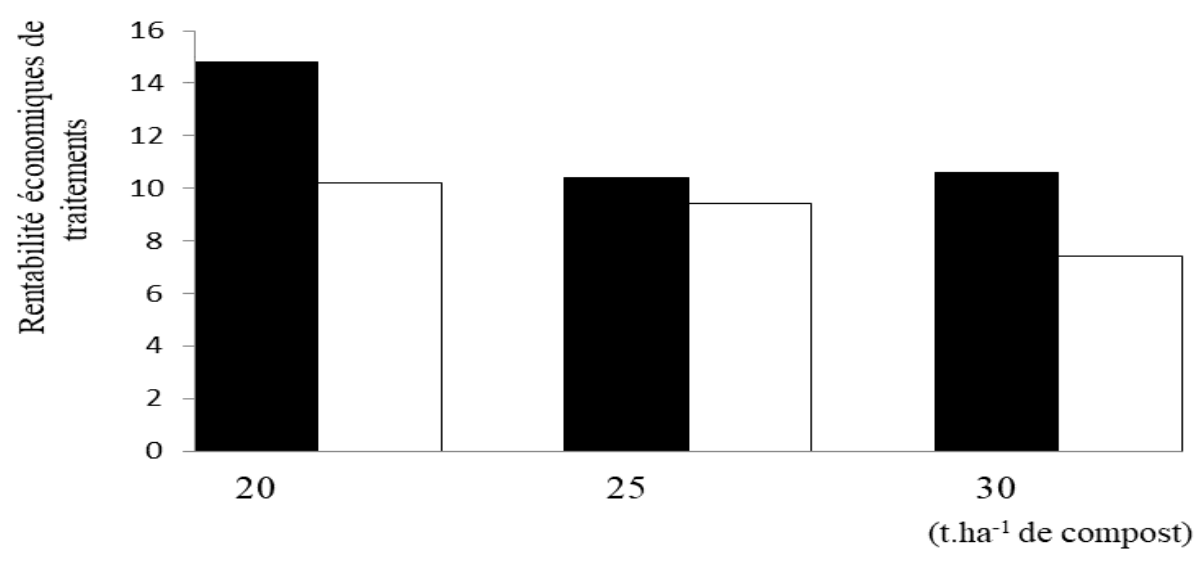

Légende : Le blanc correspond à l'ajout d'un supplément de 250kg NPK.

Figure 6. Rentabilité économique des doses de composts. 


\section{DISCUSSION}

\section{Variabilité des facteurs affectant l'efficacité agronomique des composts}

Kolwezi n'a pas un siècle d'existence, mais elle a une population estimé à ce jour à environ 1.078.568 habitants (Kanene, 2017), on assiste donc à une (péri) urbanisation dont la conséquence est l'accroissement de la ville, pourtant, selon certains écrits la naissance des quartiers spontanés serait l'une des causes et/ou des facteurs qui expliquent l'abondance de déchets à Kolwezi. Toutefois, ces déchets sont composés en grande partie des épluches des feuilles des maniocs, d'amarante et autres légumes utilisés comme supplément des repas en raison des habitudes alimentaires, expliquant le taux élevé de la part biodégradable obtenu. Une teneur élevée en phosphore alors que faible en azote comparativement aux observations de Mulaji (2011), El Herradi et al. (2014), Guermoud et Addou (2014), Temgoua et al. (2015) pourrait s'expliquer par la nature des matériaux composée notamment des coques vides d'arachide, coquille de chenille et coques vides de melon. Ces résultats sont parallèles aux propos de Bambara et al. (2014) selon que, plus il y a une variabilité des substrats à composter, plus le compost généré renfermerait une valeur chimique variée en éléments fertilisants, par conséquent une valeur agronomique souhaitable. Alors que pour Grey et Henry (1999), les pertes de cet élément sont improbables car le phosphore est fortement retenu par les composts de déchets urbains. Une faible teneur en potassium, mais comprise dans la gamme prescrite par la FAO (2005) et AFNOR, serait expliquée à sa forme inorganique dans le compost (Neilsen et al., 1998), par conséquent, susceptible au lessivage par les eaux d'arrosage au moment du compostage.

Les valeurs en éléments trace métalliques obtenues dans cette étude restent inférieures aux valeurs indicatrices de toxicité dans les sols agricoles selon la norme congolaise et internationale. Ces éléments proviendraient des légumes produits sur des substrats contaminés en ETM et arrosés aux eaux contaminées également (Atibu et al.,
2013), surtout que l'exploitation industrielle ainsi qu'artisanale reste l'une des activités économiques les plus prépondérantes de la zone d'étude.

Effets des composts ménagers sur les propriétés des sols des deux sites maraîchers et conséquences sur la productivité des cultures légumières

L'acidité du sol, qui est restée élevée sur le témoin alors qu'elle baisse aux apports de compost, serait attribuable aux bases échangeables $\left(\mathrm{Ca}^{2+}\right.$ et $\left.\mathrm{Mg}^{2+}\right)$ apportées par le compost (Yé, 2007). Selon la même source, ces bases fixées sur le complexe réduisent un nombre important d'ions acidifiants tels que $l^{\prime} \mathrm{Al}^{3+}$ et $\mathrm{H}^{+}$. Par contre, l'apport de compost, même à fortes doses, n'a pas empêché la déficience en potassium sur les deux sites. Le potassium par sa forme inorganique apporté par le compost serait facilement lessivé (Neilsen et al., 1998) ou probablement assimilé par la plante (Kettler, 2000).

Les fortes teneurs de phosphore et azote obtenues à la fin de l'expérimentation attestent que la minéralisation s'est faite au fil du temps, d'où l'intérêt d'exploiter les effets résiduels (Neilsen et al., 1998). Par contre, la difficulté à rehausser la teneur en carbone du sol, telle qu'observée dans nos essais, dénote d'une faible séquestration du carbone en zone tropicale attribuable aux pertes intenses lors de la minéralisation des matières organiques (Vagen et al., 2005; Koulibaly et al., 2015). Des taux de variations positifs observés pour l'azote, le phosphore, le pH ainsi que le rapport $\mathrm{C} / \mathrm{N}$ confirment la capacité du compost utilisé à restaurer la fertilité des sols d'étude par sa richesse en nutriment. Ces résultats sont accord avec ceux de Mulaji (2011), Nyembo et al. (2014) et Mukalay (2016) qui ont confirmé la capacité du compost à restaurer les propriétés d'un sol acide.

Quarante-cinq jours après transplantation, le compost n'a induit aucune variation significative sur la taille et le diamètre de plants. Cette inefficacité serait due à la forme organique de l'azote apportée par le compost (Matondo et Miambi, 1990). 
Ainsi, sa transformation à la forme minérale n'a pas coïncidé avec les besoins, ce qui justifierait le taux important de l'azote à la fin de l'expérimentation (Houot et al., 2004). L'amélioration de la fructification, quelle qu'en soient les doses considérées serait attribuable au rôle du phosphore libéré par le compost. Ces résultats corroborent ceux de Matondo et Miambi (1990) sur l'aubergine, (Nzuki et al., 2011) sur la tomate et (Kitabala et al., 2017) sur le gombo, qui ont montré que le phosphore libéré par les composts améliore la fructification des plantes légumières. Selon Koulibaly et al. (2015), une bonne association du phosphore et de l'azote améliorerait la fructification et le corollaire serait l'augmentation du rendement.

Les rendements moyens de tous les traitements à base de compost sont dans la gamme de rendement de la région tropicale (5 à 20 t.ha $^{-1}$ ) après usage des engrais chimiques (De Lannoy, 2001). Cette performance pourrait s'expliquer par la valeur fertilisante très hétérogène en $\mathrm{P}, \mathrm{K}$ et autres éléments minéraux fournis aux plantes par le compost durant la période expérimentale. Pour Serémé et Phal (2007), le compost ménager permet d'augmenter le rendement de culture comparativement au témoin, ce qui corrobore nos résultats et ceux d'autres chercheurs ailleurs (Matondo et Miambi, 1990; Kettler, 2000; Mulaji, 2011; Tahraoui, 2013). De matière générale, l'efficacité du compost est proportionnelle à la dose traduisant que les fortes doses ont mis à la disposition des plantes plus d'éléments nutritifs; cela rejoint les résultats obtenus par Annabi et al. (2009) et Sawadogo et al. (2008).

Quant à la réponse variétale, des différences de rendements observées seraient probablement dues au potentiel génétique (Mpika et al., 2015), néanmoins, il sied d'insister sur le fait que le rendement exprimé par ces deux variétés dans nos conditions d'étude serait expliqué en grande partie par l'usage de compost ; et cela est observable lorsque l'on compare les rendements des variétés utilisées sous les parcelles amendées à celles non amendées. Pour cette raison, nos observations permettent d'affirmer que les possibilités intrinsèques de production des plantes ne sont pleinement assurées qu'en garantissant au végétal toutes les conditions optimales de sa nutrition (Akanza et al., 2015). Déjà, Alley et Vanlauwe (2009) préconisent qu'une gestion intégrée de la fertilité des sols devra s'appuyer sur l'utilisation des germoplasmes améliorés, l'utilisation des engrais minéraux et la bonne gestion de la matière organique du sol.

L'accroissement des rendements à Musompo, pourtant un sol sableux, dénote une bonne réponse de celui-ci après les amendements organiques (Sawadogo et al., 2008) malgré qu'une différence sur le rendement n'ait pas été mise en évidence entre ces deux sites maraîchers. Par contre, la texture à dominante limoneuse constitue des conditions favorables au développement de la battance et à la dégradation de la structure des sols du périmètre maraîcher de Mwangeji. Sur ce site, cela aurait conduit à la destruction de la biomasse microbienne en partie par séchage (El Herradi et al., 2014). Une autre explication est que la matière organique serait très labile, par conséquent, susceptible d'être minéralisée rapidement. D'où, une réaction d'antagonisme d'éléments sur le site Mwangeji, pourrait justifier le faible rendement en dépit de sa relative richesse chimique. En effet, l'abondance de certains éléments fertilisants ( $\mathrm{N}$ et $\mathrm{P}$ surtout) peut générer un effet dépressif sur la croissance et le rendement de la tomate notamment.

Les composts d'ordure ménagère, des (bons) substituts des engrais minéraux

Des différences significatives expliquent qu'il existe une synergie avec l'apport complémentaire de l'engrais chimiques aux composts sur le rendement et à tous composants de rendement. Ces résultats dénotent une bonne réponse du sol aux engrais minéraux, surtout après les amendements organiques (Sawadogo et al., 2008). De ces résultats, nous pensons que l'ajout des engrais minéraux aurait augmenté la solubilité des minéraux contenus dans les matières organiques. Plusieurs auteurs ont montré que la combinaison des engrais organiques et 
minéraux crée des meilleures conditions de production par le fait que la matière organique améliore les propriétés physico-chimiques et biologiques du sol (Lompo et al., 2009) alors que les engrais minéraux apportent aux plantes les éléments nutritifs qui leur sont nécessaires pour accroitre l'efficacité agronomique (Mukengere, 2010).

Avec 30 t.ha ${ }^{-1}$ de compost, le rendement en fruit de tomate est de 11,4 contre 11,7 t.ha $^{-1}$ de rendement moyen dans la parcelle fertilisée au compost avec un ajout complémentaire des engrais chimique; ce qui signifie que l'on peut produire de la tomate avec les composts des déchets ménagers sans la fumure minérale. Le compost de déchets ménagers est donc une source d'éléments nutritifs, car il remplisse un rôle reconnu aux fertilisants. L'apport régulier serait un meilleur moyen de restaurer la fertilité des sols et d'accroître les rendements des plantes cultivées.

\section{Rentabilité de l'emploi des composts en culture de tomate et implications potentielles}

Bien que tous les traitements aient été rentables, la rentabilité est sensiblement améliorée avec l'usage du compost seul; l'apport complémentaire des engrais minéraux se traduit par une réduction de la marge bénéficiaire. Ces résultats s'expliqueraient par le fait que les charges supplémentaires d'achat des engrais ne sont pas compensées, montrant que le coût élevé des engrais chimiques serait la condition économique limitant pour une bonne performance économique en production de la tomate dans les conditions locales. Nos résultats sont parallèles à ceux de Nzuki et al. (2011) et Lumpungu et al. (2016) qui montrent que plus le coût de production était moindre, plus rentable étaient les traitements. L'usage de la faible dose soit 20 t.ha $^{-1}$ serait recommandable non seulement par la productivité induite mais, il en résulte une dose qui se traduit par une réduction du coût de production sachant que les maraîchers cherchent de la valeur ajoutée pour faire vivre leur famille. Pour la FAO (2005) et Kelly et Murekezi (2000) un agriculteur ne sera motivé d'utiliser les engrais minéraux ou organiques que dans le cas où le profit potentiel de l'investissement est le double du coût de l'investissement.

\section{Conclusion}

Le but de cette recherche était d'évaluer l'impact du compost sur la fertilité et le rendement en production légumière à Kolwezi. Cinquante-trois pourcent de la part biodégradable que renferme une poubelle d'un ménage à Kolwezi laisse estimer que le compostage pourrait être un jalon pour une meilleure gestion de déchets de la ville pourtant jusqu'ici non exploitées. Par ses composantes minérales, le compost issu de déchets ménagers parait être une réserve d'éléments nutritifs qui comblerait le besoin des plantes en légumi-culture, alors que les analyses pédologiques ont révélé la pauvreté des sols étudiés, ce qui parait mettre en évidence les faibles rendements obtenu dans toutes les parcelles non amendées. L'acidité du sol, qui est restée élevée sur le témoin alors qu'elle baisse sur les parcelles amendées, suggère le recours au compost afin de corriger le $\mathrm{pH}$. Cette correction s'est traduite par l'amélioration de la disponibilité des nutriments et par conséquent, des meilleurs rendements. L'application complémentaire d'engrais chimiques aux composts ménagers, a induit les effets significatifs uniquement avec la dose de 20 et 25 t.ha $^{-1}$ de compost. Par contre, des rendements compétitifs (11,4 contre 11,7 t.ha $^{-1}$ ) ont été obtenus avec 30 t.ha ${ }^{-}$ ${ }^{1}$ et à l'application complémentaire des engrais minéraux à la dose de 30 t.ha $^{-1}$ de compost, fait qui suggère que ce dernier accroitrait le stock d'éléments nutritifs et pourraient mieux intégrer et substitué les engrais chimiques en maraîchage. Tous les traitements ont présenté un rapport supérieur à 2, donc ils sont tous économiquement rentables. L'usage de la faible dose soit 20 t.ha ${ }^{-1}$ serait souhaitable non seulement pour la productivité induit mais, il en résulte une dose qui se traduit par une réduction du coût de production dans le contexte où beaucoup de maraîchers visent de la valeur ajoutée pour faire vivre leur famille. Pour les recherches futures, il serait intéressant de tester son efficacité sur des 
cultures autres que la tomate et de réaliser des analyses microbiennes pour une meilleure valorisation.

\section{CONFLIT D'INTERETS}

Les auteurs déclarent n'avoir aucun conflit d'intérêt.

\section{CONTRIBUTIONS DES AUTEURS}

Tous les auteurs ont participé à l'étude et à la rédaction du manuscrit. Ils ont amandé le présent article lors de son élaboration.

\section{REFERENCES}

Akanza KP, Sanogo S, N'Da HA. 2016. Influence combine des fumures organiques et minérales sur la nutrition et le rendement du maïs : impact sur le diagnostic des carences du sol. Tropicultura, 32(2): 208-220. www.tropicultura.org/text/v34n2/208.pdf

Ahmed AS, Ali M. 2006. People as partners: Facilitating people's participation in public-private partnerships for solid waste management. Habitat International, 30:781-796.

Alley MM, Vanlauwe B. 2009. The role of fertilizer in integrated plant nutrient management. IFA and TSBF-CIAT, Paris, France, 59p.

Annabi M, Bahri H, Latiri K. 2009. Statut organique et respiration microbienne des sols du nord de la Tunisie. Biotechnologie Agronomie Société Et Environnement, 13 (3) : 401-408.

Atibu KE, Devarajan N, Thevenon F, Mwanamoki PM, Tshibanda JB, Mpiana PT, Prabakar K, Mubedi JI, Wildi W, Poté J. 2013. Concentration of metals in surface water and sediment of Luilu and Musonoie Rivers, Kolwezi-Katanga, Democratic Republic of Congo. Applied Geochemistry, 39: 26-32. DOI: 10.1016/j.apgeochem.2013.09.021

Baboy LL, Kidinda KL, Tshipama TD, Tombo JA, Tshijika IM. 2015. Valorisation agricole des déchets comme alternative à leur gestion dans les villes d'Afrique subsaharienne : caractérisation des déchets urbains à Lubumbashi et évaluation de leurs effets sur la croissance des cultures vivrières. Afrique Science, 11(2) : $76-84$.

Bambara D, Bilgo A, Hien E, Masse D, Thiombiano A, Hien V. 2014. Influence des composts de déchets urbains sur les rendements du sorgho en zone soudanosahélienne du Burkina Faso. Revue Ivoirienne des Sciences et Technologie, $24: 148-171$.

Bevacqua RF, Mellano VJ. 1993. Crop response to sewage sludge compost: a preliminary report. California Agriculture, $\quad$ 47(3): 22-24. calag.ucanr.edu/archive/?type $=$ pdf\&artic le $=$ ca.v047n03p22

Cabala LS, Usenu SY, Sambieni KR, Bogaert J, Muyemba KF. 2017. Dynamique des écosystèmes forestiers de l'Arc Cuprifère Katangains en République Démocratique du Congo. I. Causes, transformations spatiales et ampleur. Tropicultura, $\quad 35$ (3): $\quad$ 192-202. http://hdl.handle.net/2268/228884

Compaoré E, Nanema LS, Bonkoungou S, Sedogo MP. 2010. Évaluation de la qualité de composts de déchets urbains solides de la ville de Bobo-Dioulasso, Burkina Faso pour une utilisation efficiente en agriculture. Journal of Applied Biosciences, 33: 2076-2083. www.m.elewa.org/JABS/2010/33/4.pdf

De Lannoy G. 2001. Légumes fruits en agriculture en Afrique tropicale. Direction générale de la coopération internationale, Ministère des Affaires Etrangères, du Commerce Extérieur et de la Coopération Internationale, Bruxelles, 503-513p.

Dibwe dia Mwembu D. 2006. Le travail des enfants dans les mines et carrières au Katanga Lubumbashi. Rapport des recherches effectuées durant la dixseptième session des travaux de l'Observatoire du Changement Urbain (OCU)-Coopération universitaire au développement (CUD Belgique).

El Herradi E-H, Soudi B, Naman F. 2014. Valorisation des déchets ménagers par extraction des substances humiques. Journal of Materials and Environmental Sciences, 5 (5): 1382-1389. 
Elattir H, Skiredj A, Elfadl A. 2003. Tomate, l'aubergine, le poivron et le gombo. Bulletin mensuel d'information et de liaison PNTTA, 4p. https://www.agrimaroc.net/bulletins/btta _100.pdf

FAO. 2005. Méthodes de compostage au niveau de l'exploitation agricole. Documents de travail sur les terres et les eaux. FAO, Rome, Italie, 51p. www.fao.org/tempref/agl/agll/docs/lwdp 2_f.pdf

Ghrabi A, R'houma A, Ennabli M, Picoud F. 2002. Caractérisation des déchets ménagers dans la région du Sahel de la Tunisie. EPCOWM, 260-270.

Grey M, Henry C. 1999. Nutrient Retention and Release Characteristics From Municipal Solid Waste Compost. Compost Science \& Utilization, 7(1): 4250.

DOI : https://doi.org/10.1080/1065657X.1999. 10701951

Guermoud N, Addou A. 2014. Etude et caractérisation des déchets ménagers de la ville de Mostaganem (Ouest-Algérie). Déchets Sciences et Techniques, 66 : 4550.

lodel.irevues.inist.fr/dechetssciencestech niques/docannexe/file/.../5_guermoud.pd $\mathrm{f}$

Houot S, Francou C, Vergé-Leviel C, Michelin $\mathrm{J}$, Bourgeois $\mathrm{S}$, Linères $\mathrm{M}$, Morel P, Parnaudeau V, Bissonais Y, Dignac MF, Dumat C, Cheiab A et Poitreanud M. 2004. Valeur agronomique et impacts environnementaux de compost d'origine urbaine : variation avec la nature du compost. AGREDE, Dossier de l'environnement de l'INRA $\mathrm{N}^{\circ} 25,107$ 123.

Kaho F, Yemefack M, Feujio-Teguefonet P, Tchantchaouang JC. 2011. Effet combiné des feuilles de Tithonia diversifolia et des engrais inorganiques sur les rendements du maïs et les propriétés d'un sol ferralitique au Centre Cameroun. Tropicultura, 29(1): 39-45. www.tropicultura.org/text/v29n1/39.pdf

Kanene MC. 2017. Planification et aménagements urbains enjeux, défis et perspectives. $\mathrm{La}$ rénovation des bidonvilles comment et par où commencer ? Expobeton, R.D.C, 31p.

Kelly V, Murekezi A. 2000. Réponse et rentabilité des engrais au Rwanda. Synthèse des résultats des études du MINAGRI menées par le Food Security Research Project (FSRP) et l'Initiative sur la Fertilité des Sols de la FAO. Food Security Research Project, FSRP/MINAGRI, Kigali, Rwanda, 4-9. https://ageconsearch.umn.edu/record/554 23

Kettler C. 2000. Évaluation comparative de la valeur fertilisante de deux composts d'ordures ménagères. Mémoire ès sciences (M.Sc.), Faculté des Sciences de l'Agriculture et de l'Alimentation, Université Laval, Canada, 92 p.

Kikufi BA, Lukoki LF. 2008. Aperçu de la végétation des sites miniers de Kolwezi au sud Katanga. Rev. Cong. Sci. Nucl., 23 (1): 21-32.

Kitabala MA, Mpundu MM, Baboy LL, Tshala UJ, Kalenda MA, Kasongo KA, Munikisa FR, Nyembo KL. 2017. Applications complémentaires des fumiers de poules et des engrais minéraux dans la culture de gombo (Abelmoschus esculentus L. Moench) à Lubumbashi : influence sur la croissance et le rendement. Congo Sciences, 5 (2): 163-168.

Kitabala MA, Tshala UJ, Kalenda MA, Tshijika IM, Mufind KM. 2016. Effets de différentes doses de compost sur la production et la rentabilité de la tomate (Lycopersicon esculentum Mill) dans la ville de Kolwezi, Province du Lualaba (RD Congo). Journal of Applied Biosciences, 102: 9669 - 9679. DOI: http://dx.doi.org/10.4314/jab.v102i1.1

Koulibaly B, Dakuo D, Ouattara A, Traoré O, Lompo F, Zombré PN, Yao-Kouamé A. 2015. Effets de l'association du compost et de la fumure minérale sur la productivité d'un système de culture à base de cotonnier et de maïs au Burkina Faso. Tropicultura, 33 (2): 125-134.

Lekasi JK, Tanner JC, Harris PJC. 2003, 
Cattle manure quality in Maragua District, Kenya: Effect of management practices and development of simple's methods of assessment. Agriculture, Ecosystem and Environnement, 94(3): 289-298.

DOI:

https://dx.doi.org/10.1016/S01678809(02)00037-3

Lompo F, Segda Z, Gnankambary Z, Ouandaogo N. 2009. Influence des phosphates naturels sur la qualité et la biodégradation d'un compost de pailles de maïs. Tropicultura, 27(2): 105-109. www.tropicultura.org/text/v27n2/105.pdf

Lumpungu CK, Bienayaku V, Mufwaya CK, Falasi N. 2016. La sciure de bois, un déchet à valoriser pour l'agriculture en R.D. Congo. Congo Sciences, 4(1): 5760.

Malaisse F. 1974. Phenology of the Zambezian woodland area with emphasis on the miombo ecosystem. In Phenology and Seasonality Modelling, Lieth $\mathrm{H}$ (ed). Springer Heidelberg: Berlin; 8: 269-286. DOI : https://doi.org/10.1007/978-3-64251863-8_23

Matondo H, Miambi E. 1990. Utilisation agricole des composts d'ordures ménagères dans la ceinture maraichère de Brazzaville (Congo). Tropicultura, 8 (3):

128-130.

www.tropicultura.org/index/author/mato ndo-h.html

Minja RR, Ambrose J, Ndee A, Swai IS, Ojiewo CO. 2011. Promising improved tomato varieties for eastern Tanzania. Afr. J. Hort. Sci., 4: 24-30. hakenya.net/ajhs/index.php/ajhs/article/d ownload/48/58

Mpika J, Attibayeba, Makoundou A, Minani D. 2015. Influence d'un apport fractionné en potassium et en azote sur la croissance et le rendement de trois variétés de tomate de la zone périurbaine de Brazzaville en République du Congo. Journal of Applied Biosciences, 94: 8789 8800.

DOI: http://dx.doi.org/10.4314/jab.v94i1.1

Mufind KM, Tshala UJ, Kitabala MA, Nyembo KL. 2016. Réponse de huit variétés de haricot commun (Phaseolus vulgaris L.) à la fertilisation minérale dans la région de Kolwezi, Lualaba (RD Congo). Journal of Applied Biosciences, 111: 10894-10904. DOI: http://dx.doi.org/104314/jab.v111i1.6

Mukalay MHJ. 2016. Identification et classification des sols sous les nouvelles normes et étude de bio-identification et restauration des unités dégradées dans la zone agricole du Haut-Katanga/R.D. Congo. Thèse de Doctorat en Sciences Agronomiques, Université de Lubumbashi, Lubumbashi, 255 p.

Mukengere BE. 2010. Evaluation de l'efficacité d'usage des engrais dans les sols dégradés du Sud-Kivu sur la culture $\mathrm{du}$ maïs et $\mathrm{du}$ haricot. Cas du groupement de Burhale. Mémoire, UEA, $59 \mathrm{p}$.

Mulaji KC. 2011. Utilisation des composts de biodéchets ménagers pour l'amélioration de la fertilité des sols acides de la province de Kinshasa (République Démocratique du Congo). Thèse de doctorat en sciences agronomiques et ingénierie biologique, Université de Liège- Gembloux Agro-Biotech, 220p. https://orbi.uliege.be/bitstream/2268/104 845/1/Mulaji_Kyela_Crispin\%28thèse\% 29.pdf

Neilsen GH, Hogue EJ, Neilsen D, Zebarth BJ. 1998. Evaluation of organic wastes as soil arnendrnents for cultivation of carrot and chard on imgated sandy soils. Canadian Journal of Soil Science, 78 (1): 217-225.

Nyembo KL, Useni SY, Chinawej MMD, Kyabuntu ID, Kaboza Y, Mpundu MM, Longanza BL. 2014. Amélioration des propriétés physiques et chimiques du sol sous l'apport combiné des biodéchets et des engrais minéraux et influence sur le comportement du maïs (Zea mays L. variété Unilu). Journal of Applied Biosciences, 74: 6121-6130. DOI: https://dx.doi.org/10.4314/jab.v74i1.7

Nzuki BF, Kinkwono EK, Sekle BG. 2011. Utilisation du guano comme substitut du Di-ammonium Phosphate (DAP) dans la fertilisation du soja et de la tomate en République Démocratique du Congo. 
Tropicultura, 29(2): 114-120. www.tropicultura.org/text/v29n2/114.pdf

Ognalaga M, Boussiengui-boussiengui G, Oyanadigui OPI. 2015. Contribution à la restauration de la fertilité des sols du périmètre maraîcher de l'IGAD DJAMITI (Franceville) par l'apport raisonné des amendements organiques et minéral. Journal of Animal \& Plant Sciences, 24(3): 3843-3853. m.elewa.org/Journals/wpcontent/uploads/2015/03/51.pdf

Rouessac F, Rouessac A, Cruché D. 2004. Analyse Chimique: Méthodes et Techniques Instrumentales Moderne (6 Ed). Dunod : Paris.

Sawadogo H, Bock L, Lacroix D, Zombré NP. 2008. Restauration des potentialités de sols dégradés à l'aide du zaï et du compost dans le Yatenga (Burkina Faso). Biotechnologie. Agronomie. Société. Environnement, 12(3): 279-290. https://popups.uliege.be:443/17804507/index.php?id=2573.

Serémé A, Phal M. 2007. Valorisation agricole des ordures ménagères en zone soudanosahélienne : cas de la ville de BoboDioulasso. Revue. CAMES-Série A, 05 : 47-54.

Sotamenou J. 2010. Le compostage : une alternative soutenable de gestion publique des déchets solides au Cameroun. Thèse de doctorat Université de Yaoundé II Faculté des Sciences Economiques et de Gestion, Département d'économie publique, Cameroun, 364 p.

Tahraoui DN. 2013. Valorisation par compostage des résidus solides urbains de la commune de Chlef, Algérie. Thèse de doctorat, Université de Limoges en Co-tutelle avec l'Université de Blida, Département sciences et techniques, Algérie, 224p.

Temgoua E, Ngnikam E, Dameni H, Kouedeu KGS. 2014. Valorisation des ordures ménagères par compostage dans la ville de Dschang, Cameroun. Tropicultura, 32 (1): 28-36.
Topanou KAN. 2012. Gestion des déchets solides ménagers dans la ville d'Abomey-Calavi (Bénin): Caractérisation et essais de valorisation par compostage. Thèse de doctorat en Chimie de l'environnement, Chimie des déchets, Université d'Abomey-Calavi. d'Abomey-Calavi, 194 p.

Useni SY, Baboy LL, Kanyenga LA, Assani Bin L, Mbuyi KM, Kasanda MN, Mbayo KLJ, Mpundu MM, Nyembo KL. 2014. Problématique de la valorisation agricole des biodéchets dans la ville de Lubumbashi : identification des acteurs, pratiques et caractérisation des déchets utilisés en maraîchage. Journal of Applied Biosciences, 76: 6326- 6337. DOI : http://dx.doi.org/10.4314/jab.v76i1.5

Useni SY, Chukiyabo KM, Tshomba KJ, Muyambo ME, Kapalanga KP, Ntumba NF, Kasangij KP, Kyungu KA, Baboy LL, Nyembo KL, Mpundu MM. 2013. Utilisation des déchets humains recyclés pour l'augmentation de la production du maïs (Zea mays L.) sur un ferralsol du sud-est de la RD Congo. Journal of Applied Biosciences, 66:5070-50811. DOI:

http://dx.doi.org/10.4314/jab.v66i0.9500 5

Vagen TG, Lal R, Singh BR. 2005, Soil carbon sequestration in subsaharan africa: a review. Land Degrad. Develop., 16: 5371. DOI : https://doi.org/10.1002/ldr.644

Yé L. 2007. Caractérisation des déchets urbains solides utilisables en agriculture urbaine et périurbaine : cas de BoboDioulasso. Mémoire de DEA, Université Polytechnique de Bobo-Dioulasso (Burkina Faso), 48p. 